\title{
Infrared thermography monitoring of the face skin temperature as indicator of the cognitive state of a person
}

by A. Stoynova

Technical University of Sofia, 8, Kl. Ohridski, Sofia, Bulgaria, ava@ecad.tu-sofia.bg

\begin{abstract}
The temperature of the face skin is an indirect indicator of the cognitive state of a person. An experimental setting for thermographic studies of the emotional state of humans is proposed. A cognitive task is used to induce stress in the form of frustration. Experimental Stroop tests is carried out, consisting of four parts and becoming more and more difficult over time. Experimental results are analysed for individual participants in the experiment. Different approaches are provided for the thermal images analysis and assessment of the emotional state based on the change in skin temperature in different regions of the face.
\end{abstract}

\section{Introduction}

The human body is a source of thermal radiation. It has a nonhomogeneous surface temperature distribution that changes, both in the presence of matologies and after medication taken and using, during loading, and making a substantial thermoregulation. The high sensitivity of spatial and time dynamics of temperature from various impact factors gives a wide range of possibilities to use thermography as a diagnostic method [1]. The surface temperature distribution amendment may occur at an emotional load when some conditions are created for stress arising from solving tests required mental load in a limited time. This can lead to a change in the chemical composition of the blood by producing hormones that affect the tone of the cardiovascular system and the condition of the skin vessels.

Thermal imaging devices can be used to provide a digital map of the surface temperature distribution of the body organ itself, and in this sense this devices are a complete means for human physiology characterization. An advantage in the use of infrared thermography is that information is obtained about the tissues and body organs functioning, which is not so simple to measure by other methods.

The face skin temperature is an indirect indicator of the cognitive state of a person. The physiological replication of increasing blood flow to the facial skin is triggered by the autonomic nervous system, whereby the blood is distributed between the skin and the periphery and the inside of the body [2].

Even more the human skin is almost a perfect emitter, with an emissivity approximately 0.98 . This fact is due to the outermost layer of the skin consisting of many small cavities, which absorb efficiently the energy with little reflection. The skin has got low reflectance. This allow accurate capturing of local temperature change using infrared thermography measurements. IRT successfully can be used for quantitative estimation of human functional states [3]. The most informative areas for thermographic stress diagnostics are the rectangular forehead area containing central vessels and the level of perfusion in the eye-socket region $[4,5]$.

Carrying out tests to assess the accumulated knowledge and skills from the learning of students is related to cognitive load. Therefore, the different aspects of the mental state have to take into account, when examinations are conducted using computers. This is an actual task directly related to the cognitive tests. The construction of effective cognitive systems is related to the knowledge of various aspects of mental states of users, such as cognitive load, focus attention and impact. Although many years of work have been done in this area, there are still unresolved issues of cognitive load assessment in a real-time, remote and non-intrusive monitoring largely related to the automation of the cognitive load assessment process.

The implementation of the present study is due to the fact that the results of performing the same tests in two ways - on paper and on a computer - vary significantly. The aim of the study is to support the development of cognitionaware systems assessing the internal state of students undergoing cognitive load during knowledge assessment test. A methodology is proposed for thermographic assessment of cognitive load in real time, which can minimize the influence of the subjective element. Thermographic measurements were carried out to assess the cognitive skills of students in solving problems on given topics. The results of the study were used to adapt the teaching material, depending on the knowledge needs.

\section{Methodology}

\subsection{Information objects on the surface of the human face}

For remote monitoring it is necessary to perform an unobtrusively thermographic measurement during the tests. For this purpose, it is most appropriate to examine the human face area for various reasons:

- The surface of the face of a physically healthy person has an uneven temperature distribution. 
- All sensory systems visual, audible, taste and touch sensation are presented in the area of the face.

- Different parts of the human face are manifested as very sensitive indicators of the internal state of the body and have increased sensitivity to internal and external stimuli.

Different areas of the face are united in an ROI (the region of interest) in thermography monitoring: nose surface; the area of the upper lip, the area of the forehead; the area around the eyes to the nose [2].

A thermography study is performed of side and full face view assessing the need to monitor the ear area, which is also highly temperature sensitive. Once it has been established that there is a strong positive linear correlation between the maximum temperature of the area around the eyes to the nose and the average external auditory channel temperature, the thermography monitoring of side face is rejected. Thermograms in side and full face view for one student and the correlation between the eye and ear temperatures, daily measured for a period of 25 days are shown on Fig.1. It has been noticed that when stratified data is processed with respect to individual students, a positive correlation is obtained.

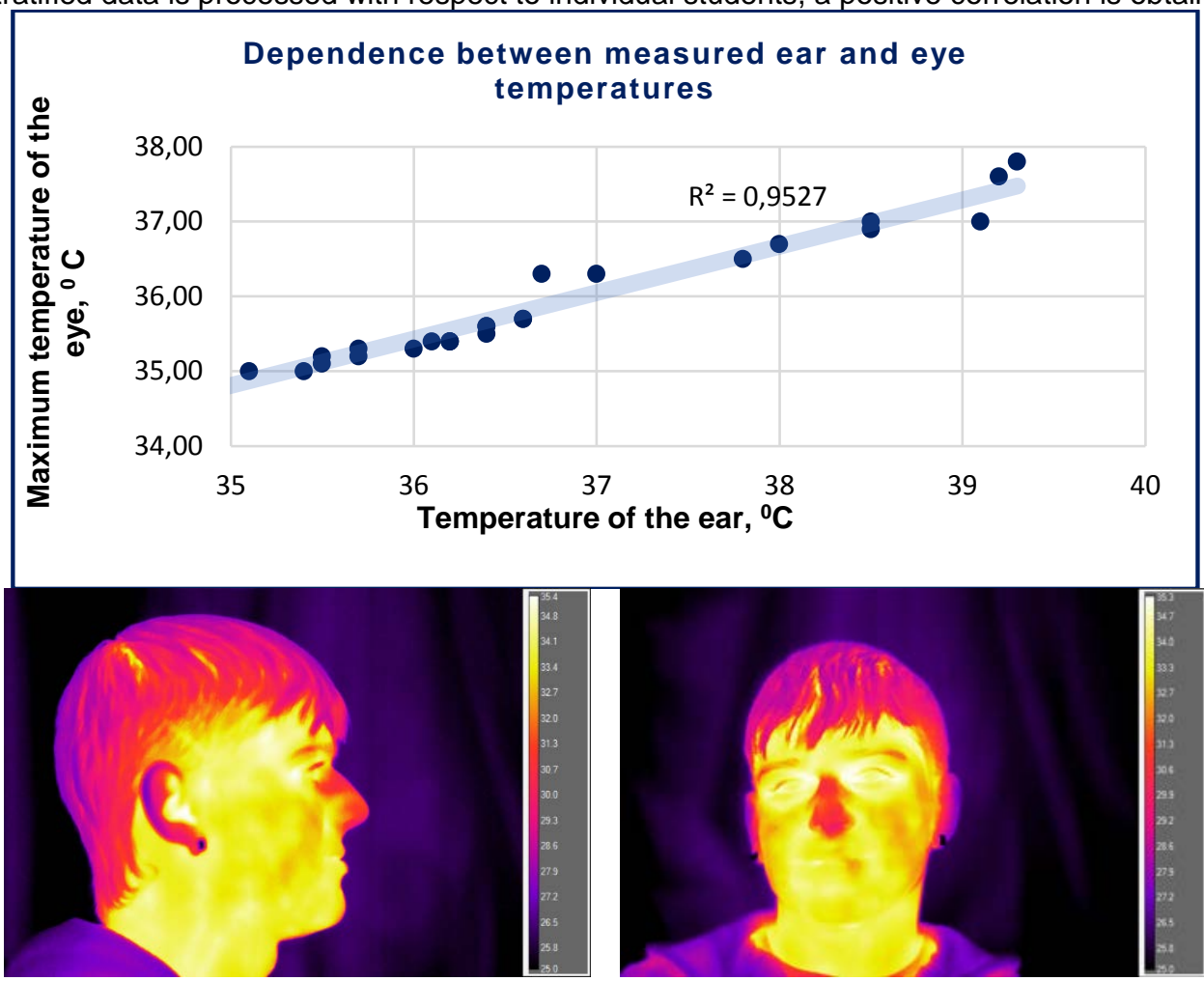

Fig. 1. Correlation between the average temperature of the external auditory channel and the maximum ocular temperature and the thermograms of side and full face view of a participant in the experiment.

The heart pulse is also measured remotely by thermographic measurement in the area of the carotid artery. The sequence of thermograms is recorded for several tens of seconds. Fig. 2 shows the line that crosses the carotid artery. The temperature profile is wavy due to the pulsations of blood flow in the vessels [3]. Using method given in research [3] the heart rate is measured remotely by infrared camera (Fig. 2). The cross-sectional dimension of the carotid artery measurement is at least 40 pixels in the thermogram. The frequency of these pulsations corresponds to the heart pulse.
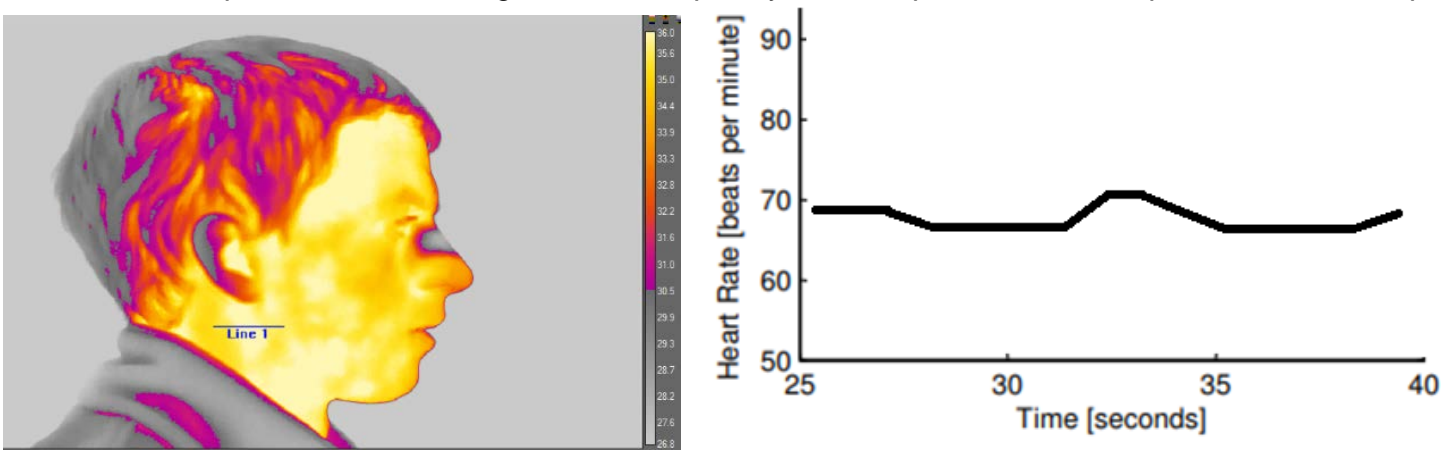

Fig. 2. Separation of the carotid artery area shown on the thermogram and measured heart rate 
Taking into account the specifics of the students participating in the experiment - students (aged 20-23), presence of glasses, beards, specific hairstyles, a ROI is selected for the study covering the central part of the forehead, nose, upper lip and the area of eyes close to the base of the nose.

A thermogram with the marked ROI on the face of a student and the thermal histograms for sequences of frames, reflecting cognitive stress during the test are shown on Fig. 3.

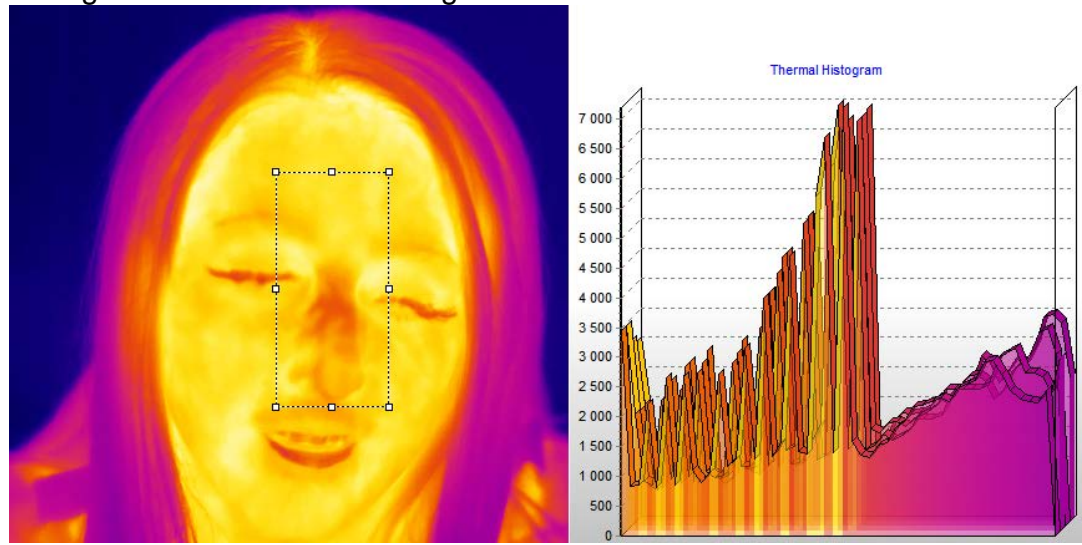

Fig. 3. Thermal histograms of the shown on the thermogram ROI for image sequences during the test

\subsubsection{Cognitive load}

Cognitive load is the amount of information the person has to process per unit of time. Individuals differ in the level of their cognitive completeness. They also differ in the amount of information they can perform the same time. When confronted with excess information, the accuracy of the information is reduced. Cognitive load depends on the environment, the individual's abilities and the desire to get information.

The cognitive loads are three types: extraneous, intrinsic and germane 6]. The optimization of the intellectual performance is possible if the cognitive load from the presented information can be assess. When a mental effort is made (for example solving difficult tasks or being tested) face temperature changes. Cognitive load is traditionally measured in two ways: by subjective self-reporting and by monitoring the user's performance in a given task or in a set of parallel tasks.

The purpose of the study is to understand, how the new form of tests influences the usefulness of applying the computer tests and adopting this form of students' examination, using thermography monitoring. The cognitive load assessment can be used to characterize all test tasks in terms of the required mental efforts of the students. Ultimately, it is good to distinguish the extraneous load from the user interface and the intrinsic load from the requirement for students' cognitive resources.

Formulated tests are categorized into three levels of objective difficulty. The easier ones included selecting a true answer from 5 options. The more difficult ones involved open questions, and the most difficult ones - solving a problem. The order of different task is balanced in terms of difficulty, with the objective of monotonically increasing and reducing the objective difficulty within the test tasks for each student. The average cognitive load during each step of the tasks is evaluated by considering the average response time (MTR). Data for the average response time (RTR) for given right answer and the average response time (RTW) for given wrong answer are also collected.

The participants in the presented study were 72 students. There were 18 women and 54 men. The participants are divided into 6 groups and each group is divided into two subgroups of 6 students. The test consists of three parts, each involving 4 questions. The first part includes questions with 5 possible answers. When someone gives a wrong answer, penalty points are given, which increases stress. The second part of the test includes questions for logical thinking, and the third part is a test for assessing analytical and creative thinking.

\subsubsection{Experimental setup}

All necessary considerations in the thermographic measurements of the human body are taken into account [10].

The measurements were conducted in a computer laboratory with air conditioning. The ambient temperature at which the tests are carried out during the different days is between $20^{\circ} \mathrm{C}$ and $22^{\circ} \mathrm{C}$ and is maintained at an accuracy of $\pm 1^{\circ} \mathrm{C}$. Measurements are performed with ThermaCam FLIR SC 640. On the Fig. 4 the view of the software during the test is shown. The infrared camera for faces monitoring is located at the level of the computer monitor. To measure the stability of the camera during the experiment, the temperature of a stable target heat source was also measured in the background of captured sequences of infrared images. To connect a computer for recording infrared sequences at high speed, a FireWire connection is used together with SDK 2.6 software. All radiometry parameters are set up for correction prior to recording. The selected video capture is 30 frames/s at size $640 \times 460$ pixels with $45^{\circ}$ lens. A compensation of the random motion of the face was undertaken on all thermal image sequences using a post-processing module. A compensation of the random motion of the face to ensure accurate and consistent positioning of measurement tools defining regions of interest in subsequent thermal image analysis was undertaken on all thermograms in the sequences using a postprocessing module. 


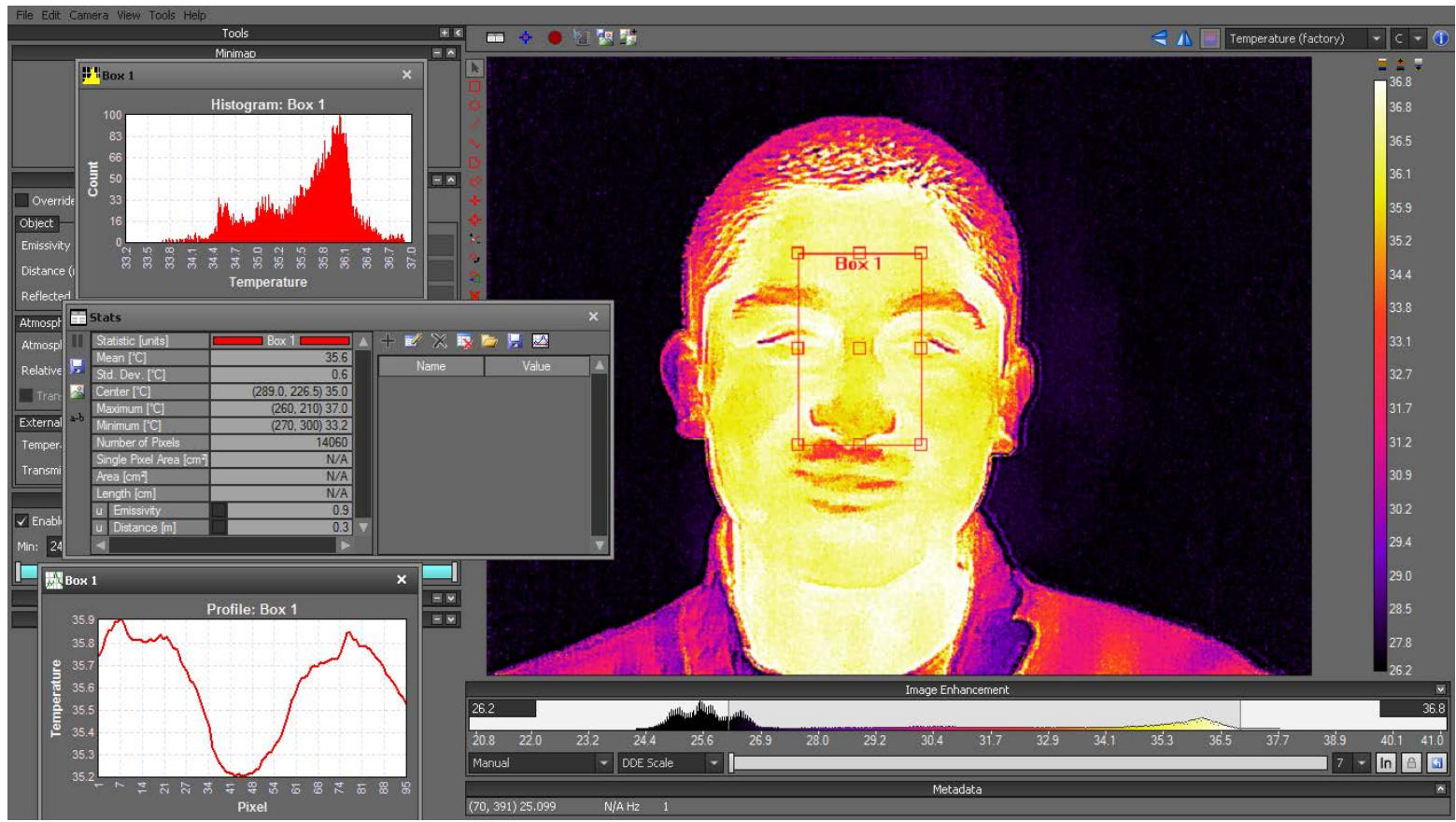

Fig. 4. Face online monitoring during test for cognitive state studying

In advance, each student study the special instructions for conducting the experiments. To determine a prestress condition, a 10-minute recording of the personal thermal images in a calm state of the students is performed before a test.

The maximum allowable test time is set using the electronic distance learning system before experiment to be started. This system takes into account the response time and gives additional statistical information on the time of access to each question in the test.

The methodology of this research includes the following steps:

- Preparatory stage - calibration and adjustment of the thermographic equipment, provision of test procedure conditions and familiarization of the participants with the instructions for conducting the test)

- Real measurement - thermography measurement before the test is started; recording participants' tests results parameters, environmental parameters during the test and thermal image sequences

- Processing and analysis - compensation of involuntary movements of the head, comparison of the test results with the thermal variations for the selected face ROI, estimation of the cognitive load using heat histograms.

\section{Results and analysis}

The histogram building process is a global operation and therefore does not depend on the geometric characteristics of the infrared images. The thermal histogram is useful for continuous observation and analysis of temperature changes of a selected object. For this purpose, an AGE environment algorithm has been developed to analyse digital thermograms and extract the temperature information at the pixel level. The main menu of the software for thermal histograms and 3D temperature models is shown on Fig. 5. The mathematical model allows to derive information about the pixel temperature levels in all areas of the thermogram, as well as to give a good visual representation of the thermogram. The software allows detailed observation of selected parts of the objects after choosing "Show Selection Area".

$\mathrm{ROI}$ is selected after marking a graphic subarea from the thermogram which can be increased. This applies when the temperature information around ROI is too heterogeneous (very hot and very cold pixels are often found at short distances). This would cause undesirable disturbances and distortions in the thermal histogram and 3D temperature model. 


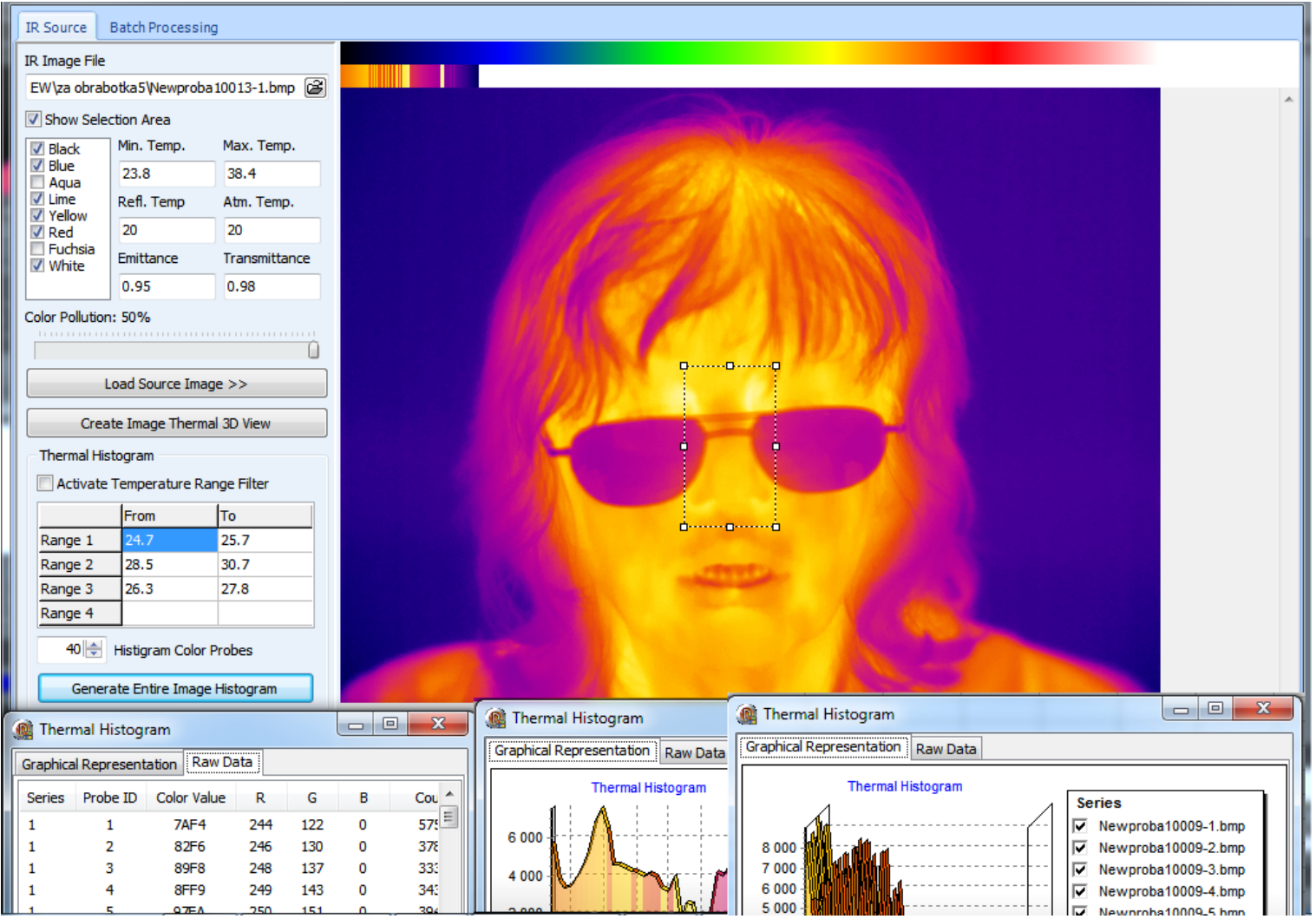

Fig.5. View of the software for thermal histogram analysis

The selection up to 4 temperature ranges and to activate 'Activate Temperature Range Filter' mode is an additional option. Thus, some pixels can be excluded from a selected ROI, for example the pixels of glasses (or moustache or hair), shown on Fig. 5. It is also possible to determine which ROI parts have the greatest temperature change during the test. The construction of thermal histograms for the thermographic sequences is a quantitative and good visual tool for the stress evaluation during the test.

The blood pressure and heart pulse are measured by the methods illustrated in Fig. 1 and Fig. 2. A strong positive correlation was found between the heart rate and the maximum surface temperature of the ROI during the test $\left(R^{2}=0.84\right)$. On Fig. 6 are shown a 3D thermogram, thermal histogram for the whole temperature range of ROI and 2D thermogram after head movement correction. The top row of pictures are results at the beginning of the test, and the bottom row - at the end of the test.

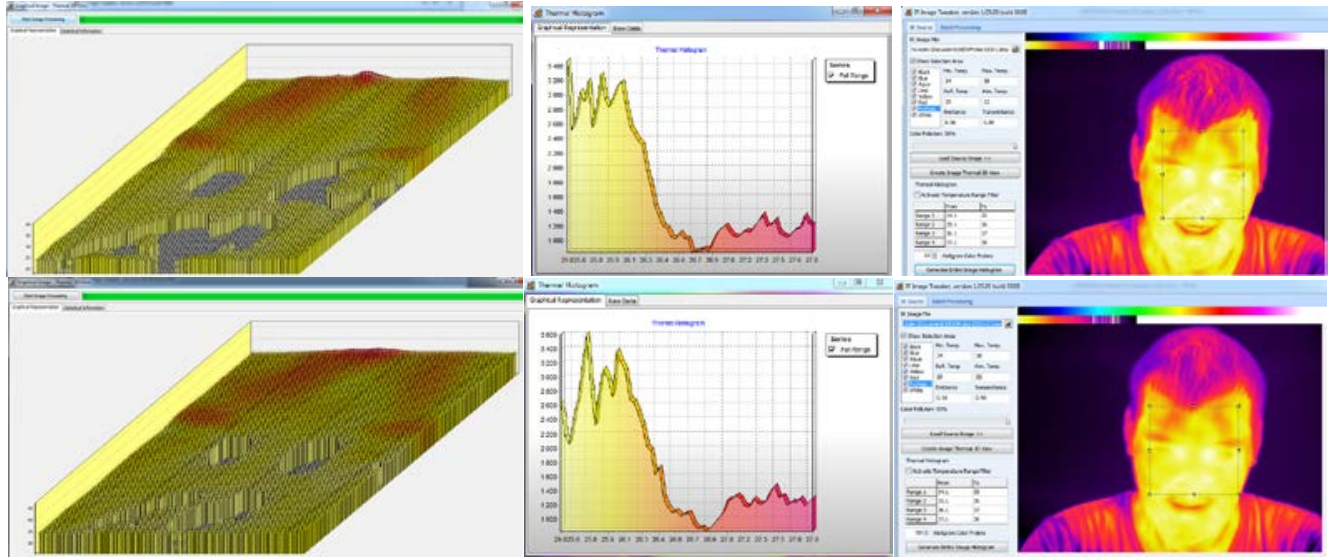

Фue.6. $3 D$ image, thermal hystogram of the ROI shown of the thermogram. Results on the up row are received before test and the result after test are shown on down row.

Thermogram and histogram sequences for one student are shown on Fig.7. The head movement is corrected on the shown termogram . On the same figure, from the left to the right, the histogram sequences obtained in the Temperature Range Filter mode for the three parts of the test are shown. It can be seen that the load (stress) from the different parts of the test is different. 


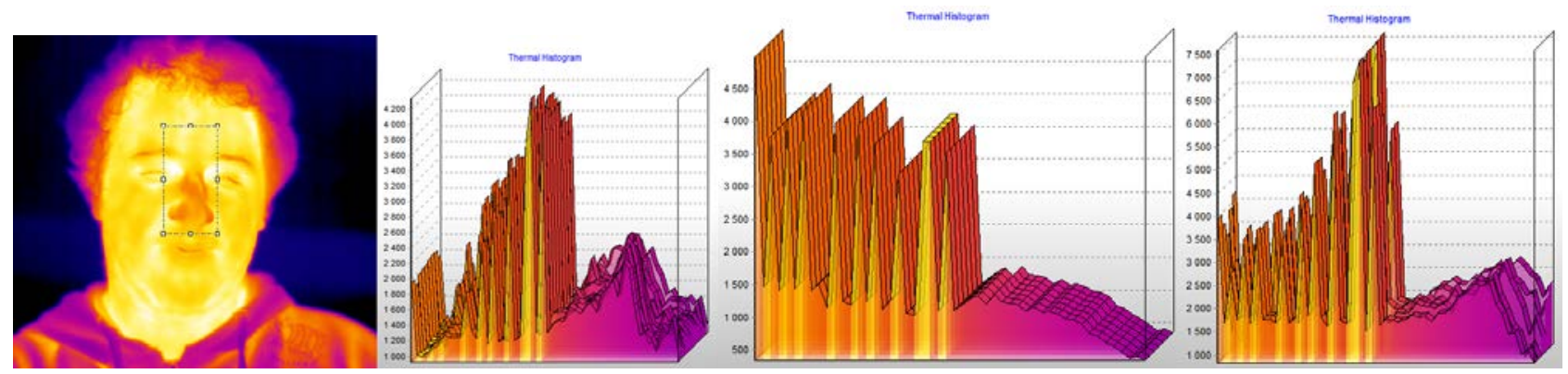

Fig.7. Thermal histograms of one student for different cognitive loads

The time for solving the first part of the test from 6 students in one subgroup is shown on Fig.8a. As stated, the first part of the test includes 4 questions with increasing difficulty. Results from the full test for a group of 12 students are shown on Fig 8b.
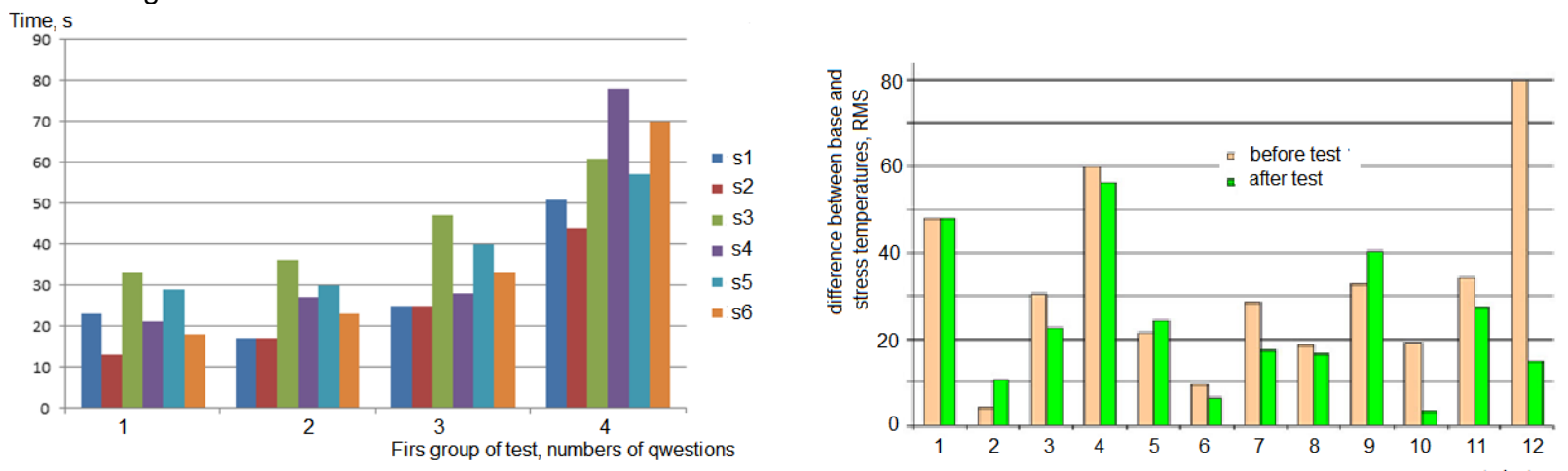

a)

Fig.8. Results from the tests for different participants

\section{Conclusions}

An approach is presented for assessing cognitive load with thermal histograms. It is shown that infrared thermography can be a powerful tool in stress level evaluation, particularly during tests.

A correlation between the face surface temperature and the results of the test was found. For participants, the correlation coefficient is $\mathrm{R}^{2}=0.82$.

Obtaining quantitative assessments of the level of stress in conducting computer tests is helpful in helping optimize tests by different criteria.

\section{Acknowledgements}

This research was funded by grant (No. DN 17/16) from the National Science Fund of Bulgaria.

\section{REFERENCES}

[1] Cardone D., Pinti P., Merla A., Thermal infrared imaging-based computational psychophysiology for psychometrics, Computational and Mathematical Methods in Medicine, article ID 984353, 1-8, 2015.

[2] St-Laurent L., Prévost D., Maldague X. "Fast and accurate calibration-based thermal/colour sensors registration", Proceedings of 10th Quantitative InfraRed Thermography conference, paper QIRT2010-126 Québec (Canada), 2010.

[3] Pavlidis I., Levine,J., Monitoring of periorbital blood flow rate through thermal image analysis and its applications to polygraph testing, 23rd Annual International Conference of the IEEE Engineering in Medicine and Biology Society, 25-28 Oct., Istanbul, Turkey, vol. 3, pp. 2826-2829, 2001.

[4] Puri C., Olson L., Pavlidis I., Levine J., Starren J., StressCam: Non-contact measurement of users' emotional states though thermal imaging, $\mathrm{CHI} 2005$ : Extended abstracts on human factors in computing systems, pp. 1725-1728, 2005.

[5] Pavlidis. I, Dowdall. J, et al, Interacting with human physiology, Computer Vision and Image Understanding, Vol 108, Pages 150-70p 2007.

[6] Puri C., Olson L., Pavlidis I., Levine J., Starren J., StressCam: non-contact measurements of users' emotional states through thermal imaging, Conference for computer-human interaction (CHI), Portland, Oregon, USA, pp. $1725-1728,2005$. 